\section{Evaluación de la ingesta dietética y excreción urinaria de sodio y potasio en adultos}

\author{
KAREN CORNEJO ${ }^{1, a}$, FERNANDO PIZARRO ${ }^{2, b}$, \\ EDUARDO ATALAH ${ }^{3}$, JOSÉ E. GALGANI ${ }^{3,4,5, \mathrm{c}}$
}

\section{Assessment of dietary intake and urinary excretion of sodium and potassium in adults}

Background: Hypertension is associated with elevated sodium and low potassium intakes. The determination of sodium and potassium intake by dietary records is inaccurate, being its measurement from 24 - $h$ urine collection the reference method. Aim: To determine urinary sodium and potassium excretion in adults. To compare dietary sodium and potassium intake and their excretion from an isolated urine sample against the reference method. Material and Methods: Seventy healthy adults aged $35 \pm 8$ years with a body mass index 25 $\pm 2 \mathrm{~kg} / \mathrm{m}^{2}$ (36 women) were studied. Urine was collected over $24 \mathrm{~h}$, including an isolated urine sample taken in fasting conditions. Additionally, three 24- $h$ dietary records were performed. Results: Reported sodium and potassium intake was 2,720 \pm 567 and 1,068 $\pm 433 \mathrm{mg} /$ day, respectively. In turn, urinary excretion of sodium and potassium was 4,770 $\pm 1,532$ and 1,852 $\pm 559 \mathrm{mg} /$ day, respectively. These latter values were significantly higher than those obtained by dietary records. Furthermore, the urinary sodium and potassium excretion estimated from an isolated urine sample was 4,839 $\pm 1,355$ and 1,845 \pm 494 $m g /$ day, respectively. These values were similar to those obtained with a $24 \mathrm{~h}$ urine collection. Conclusions: Dietary records underestimated electrolyte intake when compared with the reference method. Using an isolated urine sample to estimate electrolyte intake may be a reliable alternative.

(Rev Med Chile 2014; 142: 687-695)

Key words: Natriuresis; Sodium, dietary; Potassium.

\author{
'Programa de Magíster \\ en Nutrición y Alimentos. \\ Universidad de Chile. \\ 2Laboratorio de Micronutrientes. \\ Instituto de Nutrición y \\ Tecnología de los Alimentos. \\ Universidad de Chile. \\ ${ }^{3}$ Departamento de Nutrición. \\ Facultad de Medicina. \\ Universidad de Chile. \\ ${ }^{4}$ Departamento de Nutrición \\ Diabetes y Metabolismo. \\ Facultad de Medicina. Pontificia \\ Universidad Católica de Chile. \\ ${ }^{5}$ Carrera de Nutrición y Dietética. \\ Escuela de Medicina. Pontificia \\ Universidad Católica de Chile. \\ ${ }^{a}$ Nutricionista, MSc.

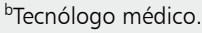 \\ 'Nutricionista, PhD. \\ Fuentes de financiamiento: \\ Fondos internos del \\ Departamento de Nutrición. \\ Facultad de Medicina. \\ Universidad de Chile (JEG). \\ Departamento de Alimentos y \\ Nutrición. Ministerio de Salud- \\ Chile (KCM) \\ Recibido el 8 de enero de 2014, \\ aceptado el 29 de mayo de 2014 . \\ Correspondencia a: \\ José E. Galgani, PhD. \\ Departamento de Nutrición, \\ Diabetes y Metabolismo. \\ Escuela de Medicina. Pontificia \\ Universidad Católica de Chile. \\ Avda. Libertador Bernardo \\ O’Higgins 340. Santiago. Chile. \\ Teléfono: 23546389. \\ Fax: 26338298. \\ jgalgani@uc.cl
}

$\mathrm{E}$ 1 Ministerio de Salud de Chile (MINSAL) indica que en nuestro país el factor de riesgo que más contribuye a la carga de mortalidad es la hipertensión arterial (una de cada siete muertes $)^{1}$. Otros estudios indican que existe una relación directa entre el consumo de sal y la presión arterial ${ }^{2-7}$. Un meta-análisis demostró que sujetos hipertensos disminuían la presión sistólica y diastólica en $\sim 4$ y $\sim 2 \mathrm{~mm} / \mathrm{Hg}$ por cada $3 \mathrm{~g} /$ día de reducción en la ingesta de sal. Una situación similar se observó en sujetos normotensos, los cuales redujeron la presión sistólica y diastólica en $\sim 2$ y $\sim 1 \mathrm{~mm} / \mathrm{Hg}$ con una reducción similar en la ingesta de sal $^{5}$. Se estimó que al disminuir la ingesta de sal en $6 \mathrm{~g} /$ día, los accidentes cardiovasculares y cardiopatías coronarias se redujeron en $14 \%$ y $9 \%$, respectivamente, en individuos hipertensos ${ }^{8}$. Basados en esta y otras evidencias, el Comité de Expertos de la Organización Mundial de la Salud (OMS) recomendó una ingesta de sodio en adultos menor a $2.000 \mathrm{mg} /$ día $(5 \mathrm{~g} / \mathrm{d} \mathrm{de} \mathrm{sal)})^{9}$.

Por otra parte, el potasio ha sido subestimado en la patogenia de las enfermedades cardiovasculares, aun cuando la relación de ingesta sodio/ potasio es un factor de mayor relevancia en la 
predicción de un evento cardiovascular respecto al sodio o potasio por sí solos ${ }^{10}$. Se estima que por cada unidad de aumento en la razón sodio/potasio, el riesgo de padecer una cardiopatía coronaria o accidente cerebrovascular aumenta $24 \%{ }^{10}$. En efecto, las guías alimentarias de Estados Unidos de Norteamérica recomiendan una ingesta de potasio en adultos de $4.700 \mathrm{mg} /$ día $^{11}$.

En Chile, la Encuesta Nacional de Salud 2010 (ENS), estimó (con muestra de orina aislada) una ingesta de sal de 9,8 g/día $(3.920 \mathrm{mg} /$ día de sodio $)^{12}$. Esta cifra es el doble de la recomendación de la OMS. La ingesta de potasio estimada con datos de excreción urinaria de la ENS fue de $2.700 \mathrm{mg} / \mathrm{día}^{13}$, cifra equivalente a la mitad de lo recomendado por las guías alimentarias de Estados Unidos de Norteamérica ${ }^{11}$.

El objetivo de este estudio fue comparar la ingesta de sodio y potasio mediante: i) excreción urinaria de sodio y potasio en $24 \mathrm{~h}$; ii) muestra aislada de orina (similar a ENS) y iii) encuesta alimentaria por recordatorio de $24 \mathrm{~h}$ en adultos.

\section{Material y Métodos}

\section{Sujetos}

Se incluyeron 70 individuos de ambos sexos con residencia en Santiago, con un índice de masa corporal (IMC) entre 20 y $30 \mathrm{~kg} / \mathrm{m}^{2}$, con peso estable en el último mes ( $<2 \mathrm{~kg}$ de diferencia) y que no reportaran cambios en el patrón alimentario y de actividad física usual. Las mediciones se realizaron entre abril y agosto de 2012. Se excluyó a los individuos en tratamiento con fármacos que modifican la función renal, también a aquellos con cuadros infecciosos, diarreas, u otras alteraciones de la salud que fuesen incompatibles con los objetivos del estudio (diabéticos, hipertensos y cardiópatas). Adicionalmente se excluyeron sujetos con indicación de modificar el consumo de sal, mujeres embarazadas y nodrizas. No fueron criterios de inclusión o exclusión el hábito tabáquico o de consumo de alcohol. El estudio fue aprobado por el Comité de Bioética de la Facultad de Medicina de la Universidad de Chile y todos los voluntarios firmaron el consentimiento informado.

\section{Diseño del estudio}

En la primera visita efectuada en el domicilio del individuo se ratificó el cumplimiento de los criterios de participación en el estudio. Luego, a los individuos elegibles de participar se les aplicó una encuesta alimentaria de recordatorio de 24 $\mathrm{h}$ y una encuesta de actividad física ${ }^{14}$. Además fueron instruidos para recolectar su orina durante $24 \mathrm{~h}^{15} \mathrm{y}$ abstenerse del consumo de alcohol en el día de recolección de la orina. Posteriormente, se realizaron dos visitas en las cuales se aplicó una encuesta alimentaria similar a la efectuada en la primera visita. Una de estas visitas se realizó un día lunes con el fin de encuestar la alimentación del fin de semana.

\section{Antropometría}

Las mediciones fueron realizadas en ayuno, descalzos y con el mínimo de ropa siguiendo procedimientos estándar ${ }^{16}$. El peso corporal se determinó con una balanza portátil (SECA 803) y la talla con un estadiómetro portátil (SECA 213).

\section{Encuesta alimentaria de 24 h y validación de la información}

Las encuestas alimentarias se realizaron en tres días distintos no consecutivos y permitió estimar la ingesta de energía, macronutrientes, sodio y potasio. Se utilizó la Tabla de Composición Química de Alimentos Chilenos ${ }^{17}$. Alternativamente se utilizó el etiquetado nutricional del alimento. Como mecanismo de validación de la información dietaria se asumió que la ingesta energética debe ser superior a 1,4 veces el gasto energético basal (GEB), por ser este el nivel mínimo de actividad física a observar en población general ${ }^{18}$.

\section{Recolección de orina}

En el día de inicio de la recolección urinaria se desechó la orina de la primera micción del día. Luego toda la orina excretada a partir de ese momento fue almacenada en un recipiente y se registró la hora de inicio. Al día siguiente, la primera orina de la mañana fue almacenada en un contenedor distinto y se registró la hora de término. La orina fue retirada ese mismo día y transportada al laboratorio para la determinación del volumen y almacenamiento a $-70^{\circ} \mathrm{C}$.

\section{Análisis de sodio, potasio y creatinina en orina}

El sodio y potasio se determinaron mediante la técnica de ión selectivo de membranas sólidas ${ }^{19} \mathrm{y}$ la creatinina mediante un método colorimétrico ${ }^{19}$. Los valores de concentración de sodio, potasio y creatinina (en $\mathrm{mg} / \mathrm{l}$ ) en combinación con el 
volumen total de orina en $24 \mathrm{~h}$ (en ml) permitió determinar la excreción total de sodio y potasio en $24 \mathrm{~h}$.

\section{Estimación de la excreción urinaria de sodio y potasio en 24 h a partir de una muestra de orina aislada}

Se utilizó la primera orina del día como muestra, en ella se estimó la excreción de electrolitos en $24 \mathrm{~h}$ mediante las ecuaciones de Tanaka et al. $^{20}$, la que incluye una corrección por creatinina urinaria. Así, la excreción estimada de sodio en $24 \mathrm{~h}(\mathrm{mg} / \mathrm{d})$ se calculó con la siguiente ecuación: $\left[\mathrm{k}_{1} \times\left(\mathrm{Na} / \mathrm{Cr}_{\mathrm{m}}\right) \times \mathrm{Cr}_{\mathrm{e} 24 \mathrm{~h}}\right]^{0,39} \times \mathrm{k}_{2}$, donde: $\mathrm{k}_{1}, 22 \mathrm{y} \mathrm{k}_{2}$, 23; $\mathrm{Na}$, concentración de sodio en muestra de orina $(\mathrm{mEq} / \mathrm{l}) ; \mathrm{Cr}_{\mathrm{m}}$, concentración de creatinina en muestra de orina $(\mathrm{mg} / \mathrm{dl})$. La creatinina en $24 \mathrm{~h}$ $\left(\mathrm{Cr}_{\mathrm{e} 24 \mathrm{~h}}\right)$ se calcula como: $\mathrm{Cr}_{\mathrm{e} 24 \mathrm{~h}}=-2 \times$ edad (años) + $14,9 \times$ peso $(\mathrm{kg})+16,1 \times$ talla $(\mathrm{cm})-224$. De forma similar, la excreción estimada de potasio en $24 \mathrm{~h}$ $(\mathrm{mg} / \mathrm{d})$ corresponde a $\left[\mathrm{k}_{1} \times\left(\mathrm{K}_{/} \mathrm{Cr}_{\mathrm{m}}\right) \times \mathrm{Cr}_{\mathrm{e} 24 \mathrm{~h}}\right]^{0,43} \times \mathrm{k}_{2}$, donde: $\mathrm{k}_{1}=7,6 \mathrm{y}_{2}=39 ; \mathrm{K}=$ concentración de potasio en muestra de orina $(\mathrm{mEq} / \mathrm{l})$.

\section{Estadística}

Los datos se expresaron como promedio y desviación estándar o mediana y rango intercuartílico según la distribución de las variables, la que se evaluó mediante el test de Shapiro-Wilk. Las diferencias entre promedios se evaluaron mediante la prueba $t$ de Student de dos colas, y las diferencias entre medianas se evaluaron mediante la prueba de Mann-Whitney. La correlación entre variables se determinó mediante test de Pearson y el análisis de concordancia entre métodos se analizó mediante el análisis de Bland-Altman ${ }^{21}$. Finalmente, las diferencias se consideraron significativas cuando el valor de $\mathrm{p}$ fue menor a 0,05 .

\section{Resultados}

Las características generales de los sujetos se presentan en la Tabla 1. Los hombres y mujeres tuvieron edades similares, mientras que los hombres presentaron mayor peso y estatura que las mujeres $(\mathrm{p}<0,05)$.

La ingesta energética reportada en ambos grupos fue $1.998 \pm 265 \mathrm{kcal} /$ día. El GEB estimado fue $1.590 \pm 221 \mathrm{kcal} /$ día y el gasto energético total mínimo estimado (GETME, i.e. GEB $\times 1,4)$ fue
$2.226 \pm 309 \mathrm{kcal} / \mathrm{d}$. En función de este análisis, se observó una adecuación entre la ingesta energética reportada y GETME de $90 \pm 11 \%$, significativamente menor al nivel de adecuación de 100\% $(\mathrm{p}<0,0001)$. La ingesta energética reportada fue mayor en hombres respecto a mujeres $(2.159 \pm 231$ vs $1.845 \pm 198 \mathrm{kcal} / \mathrm{d}$, respectivamente; $\mathrm{p}<0,01)$. Esta diferencia desapareció al corregir por el peso corporal ( $28 \pm 3$ vs $29 \pm 4 \mathrm{kcal} / \mathrm{d} / \mathrm{kg}$ peso corporal, respectivamente; $\mathrm{p}=0,16)$.

La ingesta reportada de sodio (mg/día) fue mayor en hombres respecto a mujeres $(p<0,01$; Tabla 2). Sin embargo, al ser corregido por peso corporal, no hubo diferencias atribuidas al sexo ( $\mathrm{p}=0,51$; Tabla 2). Respecto a la ingesta de potasio, no se observaron diferencias entre sexos, independiente de la unidad de expresión de los valores (Tabla 2 ).

La excreción urinaria de sodio en $24 \mathrm{~h}$ fue mayor en hombres respecto a mujeres $(\mathrm{p}<0,01$; Tabla 2) y se observó una relación directa entre la excreción urinaria de sodio y el peso corporal $(\mathrm{r}=0,69 ; \mathrm{p}<0,01 ;$ Figura 1A). La diferencia por género desapareció al corregir la excreción urinaria de sodio en $24 \mathrm{~h}$ por peso corporal ( $\mathrm{p}=0,34$; Tabla 2).

La excreción urinaria de potasio tuvo un comportamiento similar al de sodio, hubo mayor excreción urinaria en hombres respecto a mujeres $(\mathrm{p}=0,03$; Tabla 2), la cual desaparece al corregir por peso corporal ( $\mathrm{p}=0,50$; Tabla 2$)$. Asimismo, se observó una relación directa entre la excreción urinaria de potasio ( $\mathrm{mg} /$ día) y el peso corporal $(\mathrm{kg})(\mathrm{r}=0,49, \mathrm{p}<0,01 ;$ Figura $1 \mathrm{~B})$.

La ingesta dietética y excreción urinaria de sodio se asociaron de manera directa $(r=0,46$; $\mathrm{p}<0,01$. Figura 2A). Paralelamente, el análisis de la relación entre el promedio y la diferencia entre ingesta y excreción de sodio (Blant-Altman) mos-

Tabla 1. Características generales de los sujetos

\begin{tabular}{|lcc|}
\hline & Hombres & Mujeres \\
$\mathrm{n}$ & 34 & 36 \\
Edad (años) & $35 \pm 8$ & $35 \pm 9$ \\
Peso (kg) & $78 \pm 6$ & $63 \pm 5^{*}$ \\
Talla (cm) & $172 \pm 4$ & $160 \pm 4^{*}$ \\
IMC (kg/m $\left.\mathrm{m}^{2}\right)$ & $26,2 \pm 2,0$ & $25,0 \pm 1,4$ \\
\hline
\end{tabular}

${ }^{*} p<0,05$. 
Tabla 2. Ingesta y excreción urinaria de sodio y potasio

\begin{tabular}{|c|c|c|c|}
\hline & Hombres & Mujeres & $\mathbf{p}$ \\
\hline $\mathrm{n}$ & 34 & 36 & \\
\hline \multicolumn{4}{|l|}{ Ingesta } \\
\hline Sodio $(\mathrm{mg} / \mathrm{d})$ & $2.960 \pm 587$ & $2.495 \pm 448$ & $<0,01$ \\
\hline Sodio (mg/d/kg) & $38 \pm 7$ & $39 \pm 6$ & 0,51 \\
\hline Potasio (mg/d) & $1.168 \pm 443$ & $972 \pm 407$ & 0,06 \\
\hline Potasio (mg/d/kg) & $15 \pm 6$ & $15 \pm 7$ & 0,86 \\
\hline \multicolumn{4}{|l|}{ Mediciones en $24 \mathrm{~h}$} \\
\hline Sodio $(\mathrm{mg} / \mathrm{d})$ & $5.240 \pm 1.373$ & $4.069 \pm 858$ & $<0,01$ \\
\hline Sodio $(\mathrm{mg} / \mathrm{d} / \mathrm{kg})$ & $67 \pm 16$ & $64 \pm 12$ & 0,34 \\
\hline Potasio (mg/d) & $2.051 \pm 667$ & $1.745 \pm 433$ & 0,03 \\
\hline Potasio (mg/d/kg) & $26 \pm 8$ & $27 \pm 6$ & 0,50 \\
\hline Razón sodio/potasio (mg/mg) & $2,7 \pm 0,9$ & $2,5 \pm 0,8$ & 0,23 \\
\hline \multicolumn{4}{|l|}{ Estimaciones para $24 \mathrm{~h}$} \\
\hline Creatinina (mg/d) & $1.615 \pm 136$ & $1.218 \pm 144$ & 0,54 \\
\hline Sodio $(\mathrm{mg} / \mathrm{d})$ & $5.304 \pm 1.235$ & $4.003 \pm 963$ & $<0,01$ \\
\hline Potasio (mg/d) & $2.100 \pm 648$ & $1.669 \pm 402$ & $<0,01$ \\
\hline Razón sodio/potasio (mg/mg) & $2,63 \pm 0,57$ & $2,49 \pm 0,64$ & 0,35 \\
\hline
\end{tabular}

La ingesta se determinó a partir de tres encuestas de $24 \mathrm{~h}$ aplicadas a cada individuo en días no consecutivos. Los electrolitos fueron medidos a partir de la orina recolectada en un día entero por los individuos. La estimación de la creatinina en 24 h se realizó mediante la ecuación de Tanaka et al. ${ }^{20}$, la cual utiliza datos de peso, talla y edad. La estimación del sodio y potasio urinario en $24 \mathrm{~h}$ a partir de la ecuación de Tanaka et al. ${ }^{20}$, la cual se basa en la creatinina estimada en $24 \mathrm{~h}$, junto con la creatinina y electrolito respectivo medidos en una muestra de orina aislada (ayuno). El valor de creatinina en la muestra de orina aislada fue en hombres y mujeres de $526 \pm 210 \mathrm{mg} / \mathrm{dl}$ y $692 \pm 330 \mathrm{mg} / \mathrm{dl}$, respectivamente.
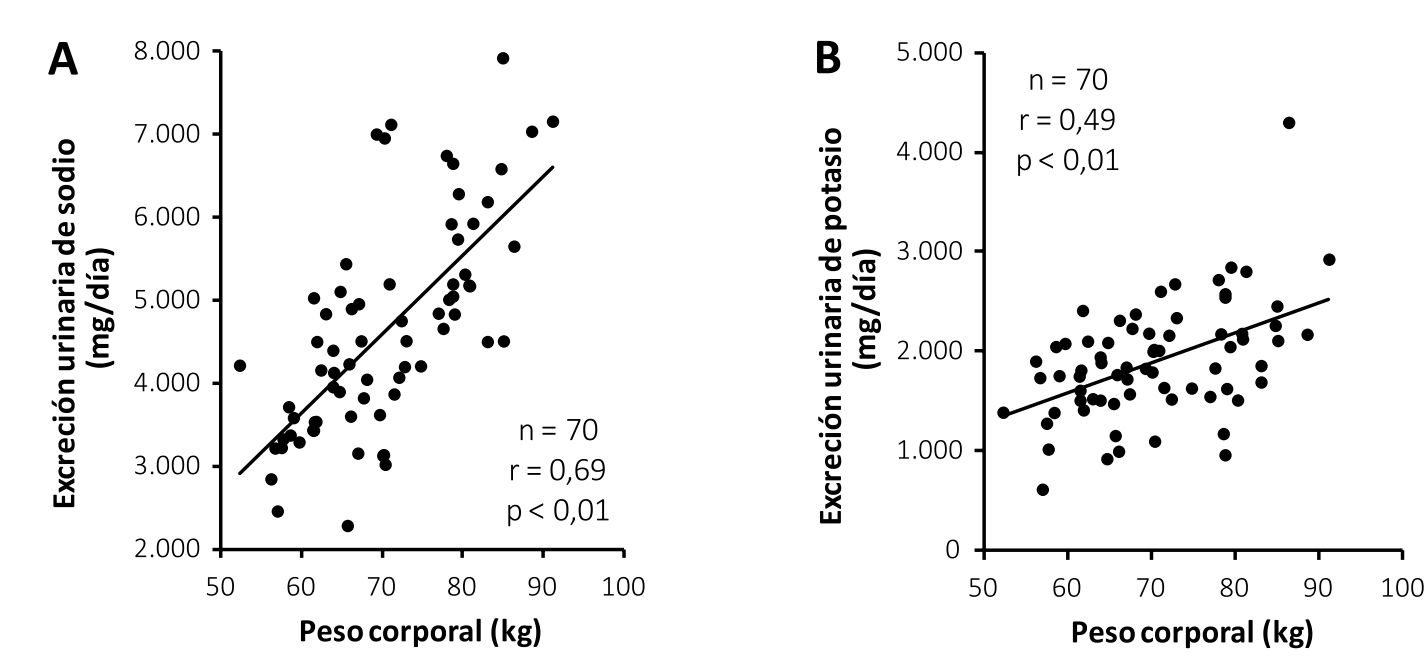

Figura 1. Relación entre peso corporal y urinaria de sodio (A) y potasio (B) en $24 \mathrm{~h}$. 

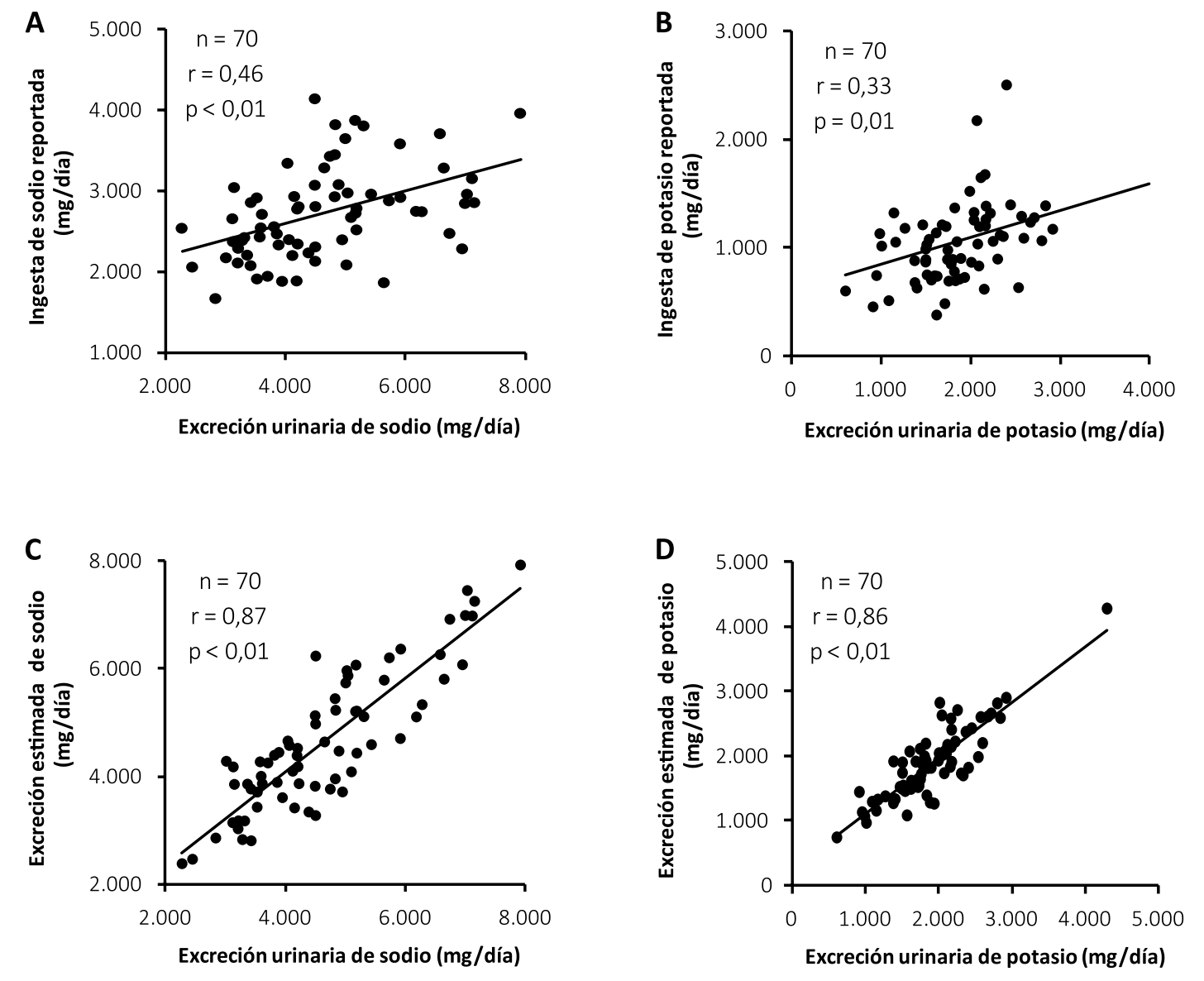

Figura 2. Relación entre indicadores de ingesta de electrolitos. Ingesta dietética y excreción urinaria de sodio (A) y potasio (B) en 24 h; y Excreción a partir de muestra de orina aislada y de $24 \mathrm{~h}$ para sodio (C) y potasio (D).

tró una línea de regresión con pendiente negativa $(-1,00 \pm 0,12 \mathrm{mg} / \mathrm{d} ; \mathrm{p}<0,01$. Figura 3A). De esta forma, a mayor nivel de excreción urinaria de sodio, mayor es la divergencia observada con la magnitud de la ingesta de sodio reportada.

Un análisis similar efectuado para el potasio, también mostró una relación directa entre la ingesta y excreción de este electrolito $(r=0,33$; $\mathrm{p}=0,01$. Figura $2 \mathrm{~B}$ ). Nuevamente, el análisis de Blant-Altman también mostró una pendiente negativa para la relación entre el promedio y la diferencia observada $(-0,42 \pm 0,17 \mathrm{mg} / \mathrm{d} ; \mathrm{p}<0,01$. Figura 3B).

La relación entre los valores de sodio medidos por excreción urinaria en $24 \mathrm{~h}$ y muestra de orina aislada fue significativa $(r=0,87 ; p<0,01$. Figura $2 \mathrm{C})$ igual que para el potasio $(\mathrm{r}=0,86 ; \mathrm{p}<0,01$. Figura 2D). Mediante análisis de Blant-Altman se observó que tanto la pendiente como el intercepto para la relación entre el promedio y la diferencia entre métodos (excreción diaria estimada vs medida) no fue distinta de cero. En el caso del sodio, el valor de la pendiente e intercepto fueron $0,00 \pm 0,06 \mathrm{mg} /$ día $(\mathrm{p}=0,98)$ y $-11 \pm 307 \mathrm{mg} /$ día $(\mathrm{p}=0,97)$, respectivamente (Figura $3 \mathrm{C})$. En el caso del potasio, la pendiente e intercepto fue- $0,00 \pm$ $0,07 \mathrm{mg} /$ día $(\mathrm{p}=0,98)$ y $-11 \pm 129 \mathrm{mg} /$ día $(\mathrm{p}=$ 0,93 ), respectivamente (Figura $3 \mathrm{D}$ ). 

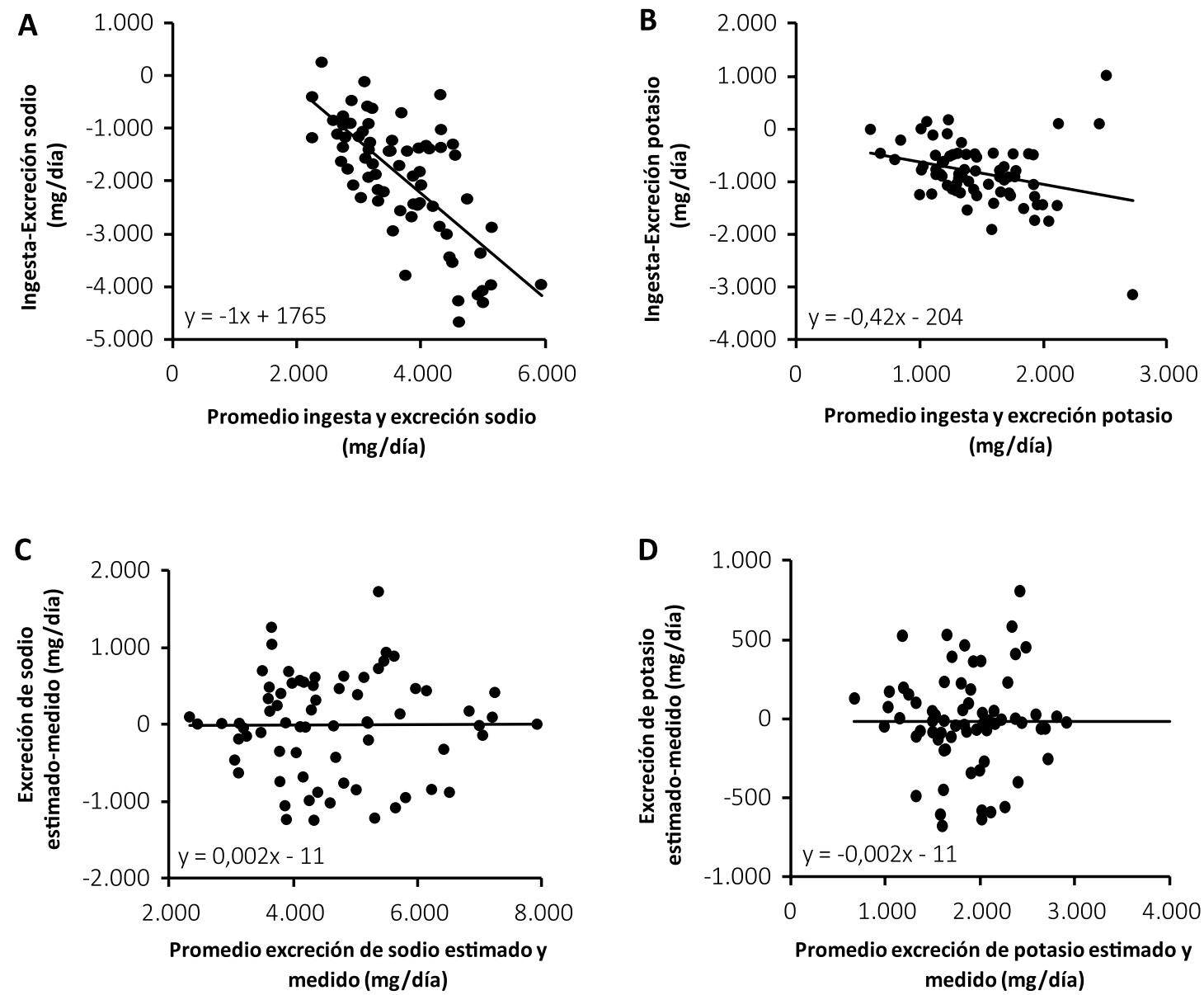

Figura 3. Concordancia entre métodos de estimación de la ingesta de electrolitos. A) Concordancia entre encuesta alimentaria y excreción urinaria de sodio en $24 \mathrm{~h}$. El eje $x$ corresponde al promedio entre la ingesta de sodio reportada y el sodio medido en orina de $24 \mathrm{~h}$. El eje y corresponde a la diferencia entre la ingesta de sodio reportada y el sodio medido en orina de $24 \mathrm{~h}$. B) Concordancia entre encuesta alimentaria y excreción urinaria de potasio en $24 \mathrm{~h}$. El eje $x$ corresponde al promedio entre la ingesta de potasio reportada y el potasio medido en orina de $24 \mathrm{~h}$. El eje $y$ corresponde a la diferencia entre la ingesta de potasio reportada y el potasio medido en orina de $24 \mathrm{~h}$. C) Concordancia entre muestra de orina aislada y de $24 \mathrm{~h}$. El eje $x$ corresponde al promedio entre el sodio estimado con la muestra y el sodio medido en orina de $24 \mathrm{~h}$. El eje y corresponde a la diferencia entre el sodio estimado con la muestra y el sodio medido en orina de $24 \mathrm{~h}$. D) Concordancia entre muestra de orina aislada y de $24 \mathrm{~h}$. El eje $x$ corresponde al promedio entre el potasio estimado con la muestra y el potasio medido en orina de $24 \mathrm{~h}$. El eje $y$ corresponde a la diferencia entre el potasio estimado con la muestra y el potasio medido en orina de $24 \mathrm{~h}$.

\section{Discusión}

En este estudio confirmamos que la ingesta de sodio y potasio estimada por encuestas alimentarias subestima la excreción urinaria de estos electrolitos. A partir de la estimación del mínimo gasto energético total a observar en población general (1,4 veces el gasto energético basal), se observó una subestimación cercana a $10 \%$ de la ingesta energética total. En función de este análisis se esperaría una subestimación de la ingesta de sodio y potasio de magnitud similar. 
Sin embargo, se encontró que la subestimación de la ingesta de sodio y potasio respecto a su nivel excretado fue alrededor de $40 \%$. Este análisis asume una absorción intestinal de los electrolitos de $100 \%$, y una excreción urinaria equivalente a su ingesta. En individuos en balance de fluidos y electrolitos, dichos supuestos tienen un error menor a $5 \%{ }^{22}$. Esta divergencia puede deberse a que la actividad física real de los individuos fue mayor al mínimo estimado. De ser ésta la única fuente de error, el nivel de actividad física real de los individuos debiera ser aproximadamente de 2,1. Esta situación es improbable, dado que en poblaciones sedentarias el nivel de actividad física promedio es cercano a $1,55^{18}$. Esto sugiere que otros factores determinan la subestimación del reporte dietético de sodio. Es probable, que la menor ingesta reportada sea consecuencia de la inexactitud del contenido de sodio reportado en las tablas de composición química de alimentos o etiquetado nutricional. Adicionalmente, puede deberse al error en la estimación de la cantidad de sal añadida a los alimentos durante la cocción o consumo de éstos. En función de este análisis, la determinación de la ingesta de electrolitos a partir de encuestas alimentarias es particularmente compleja.

En nuestro país existen pocos estudios sobre el consumo de sal en Chile. Ninguno de ellos ha estimado la ingesta a partir de la excreción urinaria en $24 \mathrm{~h}$, el cual es el método de referencia sugerido por la OMS. En este estudio observamos que la ingesta promedio de sodio fue cercana a $4 \mathrm{~g}$ por día, lo cual equivale a alrededor de $10 \mathrm{~g}$ de sal por día, siendo este valor el doble de la recomendación actual $^{23}$.

Respecto a la ingesta de potasio, no disponemos de información en nuestro país. Dado el creciente interés en la relación sodio/potasio más que el sodio por si sólo en la etiopatogenia de la hipertensión arterial, la estimación de su balance corporal se hace relevante. En este estudio la razón entre el sodio y potasio excretados en orina fue 4,5, cifra similar a 3,6 la reportada por la ENS20092010. Estos datos sugieren y a la vez confirman los resultados de estudios previos, en los cuales se constata la existencia de un patrón alimentario con bajo consumo de frutas, verduras y leguminosas, ricos en potasio.

El método de referencia para estimar la ingesta de electrolitos es la recolección de orina en $24 \mathrm{~h}$. La aplicación de este indicador representa algunos desafíos logísticos que dificultan su implementación masiva. Por ello, algunos autores han preferido utilizar alternativas más sencillas para estimar la excreción urinaria de estos electrolitos. Con este objetivo, Tanaka et al. ${ }^{20}$ propusieron una ecuación para estimar la excreción de electrolitos en $24 \mathrm{~h}$ a partir de una muestra de orina corregida por la concentración de creatinina de dicha muestra. Este método fue aplicado en la ENS $2010^{12}$, observando una excreción de sodio de $3.920 \mathrm{mg} /$ día y una razón sodio/potasio de 3,6. Mediante el mismo método, López et al. ${ }^{24}$ estimaron una excreción de sodio equivalente a $4.992 \mathrm{mg} / \mathrm{d}$ a partir de muestras de orina aislada en adultos chilenos, siendo estos resultados comparables a los observados en este estudio. Además, en el presente estudio, existió una alta concordancia entre la excreción urinaria de sodio y potasio estimada a partir de una muestra urinaria aislada respecto a orina recolectada por $24 \mathrm{~h}$.

El análisis de concordancia (Bland \& Altman) entre métodos de excreción urinaria de electrolitos en $24 \mathrm{~h}$ basados en la determinación o estimación de las variables (sodio y potasio) mostró que la diferencia entre métodos es cero. Es decir, el promedio de excreción de sodio y potasio para ambos métodos es similar. Sin embargo, dicho análisis también muestra una alta variabilidad entre individuos, lo que restringe su uso a estudios poblacionales más que individuales.

En conclusión, se confirma que la ingesta de sodio es prácticamente el doble de la recomendación actual. Por otra parte, las encuestas alimentarias subestiman el nivel de ingesta cuando se compara con el método de referencia. Más aún, el grado de subestimación aumenta en individuos con ingestas elevadas de sodio. Un hallazgo que puede tener relevancia en salud pública es la estrecha relación existente entre la medición de la excreción de sodio a partir de orina recolectada por $24 \mathrm{~h}$ y una muestra aislada. Esto puede facilitar la evaluación de la ingesta de sodio en la población, siempre que se incluya la corrección por creatinina urinaria.

\section{Referencias}

1. Ministerio de Salud de Chile, Departamento de Salud Pública, Escuela de Medicina Pontificia Universidad Católica de Chile. Estudio de Carga de Enfermedad y Carga Atribuible, julio de 2008. Disponible en: http:// 
epi.minsal.cl/epi/html/invest/cargaenf2008/Informe $\% 20$ final\%20carga_Enf_2007.pdf. [Consultado el 3 de diciembre de 2011].

2. Stamler J, Elliott P, Dennis B, Dyer A, Kesteloot H, Liu K, et al. INTERMAP: background, aims, design, methods and descriptive statistics (non-dietary). J Hum Hypertens 2003; 17 (9): 591-608.

3. Law M. Salt, blood pressure and cardiovascular diseases. J Cardiovasc Risk 2000; 7 (1): 5-8.

4. Penney S. Dropping the salt: Practical steps countries are taking to prevent chronic non communicable diseases through population wide dietary salt reduction. Public Health Agency of Canada. 2009; p.6-12. Disponible en: www.paho.org/English/AD/dpc/nc/salt-mtg-phac-paper. pdf. [Consultado el 3 de marzo de 2012].

5. He F, MacGregor G. Effect of modest salt reduction on blood pressure: a meta-analysis of randomized trials implications for public health. J Hum Hypertens 2002; 16 (11): 761-70.

6. Sacks F, Svetkey L, Vollmer W, Appel L, Bray G, Harsha $D$, et al. Effects on blood pressure of reduced dietary sodium and the dietary approaches to stop hypertension (DASH) diet. N Engl J Med 2001; 344 (1): 3-10.

7. Intersalt Cooperative Research Group. Intersalt: an international study of electrolyte excretion and blood pressure. Results for 24 hour urinary sodium and potassium excretion. Br Med J 1988; 297 (6644): 319-28.

8. He F, MacGregor G. How far should salt intake be reduced? Hypertension 2003; 42 (6): 1093-9.

9. World Health Organization. Diet, Nutrition and the Prevention of Chronic Diseases. Joint WHO/FAO Expert Consultation. Disponible en: http://www.fao.org/ docrep/005/ac911e/ac911e00.HTM. [Consultado el 26 de abril de 2012].

10. Cook N, Obarzanek E, Cutler J, Buring J, Rexrode K, Kumanyika $\mathrm{S}$, et al. Joint effects of sodium and potassium intake on subsequent cardiovascular disease: the Trials of Hypertension Prevention (TOHP) Follow-up study. Arch Intern Med 2009; 169 (1): 32-40.

11. U.S. Department of Agriculture, U.S. Department of Health and Human Services. Dietary Guidelines for Americans 2010. Disponible en: http://www.cnpp.usda. gov/Publications/DietaryGuidelines/2010/PolicyDoc/ PolicyDoc.pdf. [Consultado el 26 abril de 2012].

12. Ministerio de Salud Chile, Pontificia Universidad Católica de Chile. Encuesta Nacional de Salud ENS Chile 2009-2010. Disponible en: http://www.redsalud.gov.cl/ portal/url/item/99bbf09a908d3eb8e04001011f014b49. pdf. [Consultado el 4 de diciembre de 2011].

13. Zárate L, Valenzuela A. Equilibrio sodio-potasio en la regulación de la hipertensión arterial. Medwave 2012; 12
(2): e5301doi:10.5867/medwave. 2012.02.5301. Disponible en: www.mednet.cl/link.cgi/Medwave/Revisiones/ RevisionClinica/5301. [Consultado el 3 de mayo de 2013].

14. Booth M. Assessment of physical activity: An international perspective. Research quarterly for Exercise and sport. 2000; 71 (2): s114-120. Disponible en: http://www. ipaq.ki.se/questionnaires/SpanIQSHL7SELFrev230802. pdf. [Consultado el 5 de mayo de 2012].

15. OPS-OMS Grupo regional de expertos para la prevención de enfermedades cardiovasculares mediante reducción del consumo de sal en toda la población. Protocolo de determinación de la concentración de sodio en muestras de orina de veinticuatro horas en la población. 2010. Disponible en: http://www.paho. org/hq../index.php?option=com_docman\&task $=$ doc_ view\&gid=21515\&Itemid. [Consultado el 3 de marzo de 2012].

16. World Health Organization Methodology of Nutritional Surveillance. Physical Condition: Use and Interpretation of Anthropometric Data. Joint FAO/UNICEF/WHO Experts 1995.Disponible en: http://archive.unu.edu/ unupress/food/V182e/ch12.htm. [Consultado el 16 de junio de 2012].

17. Schmidt HH, Pennacchiotti MI, Masson SL, Mella RM. Tabla de Composición Química de Alimentos Chilenos. 1992. Disponible en: http://www.captura. uchile.cl/handle/2250/5552. [Consultado el 16 de junio de 2012].

18. FAO Food and Nutrition Technical Report Series 1. Human energy requirements.Report of a Joint $\mathrm{FAO} / \mathrm{WHO} /$ UNU Expert Consultation. Rome, 17-24 october 2001. Disponible en: ftp://ftp.fao.org/docrep/fao/007/y5686e/ y5686e00.pdf. [Consultado el 18 de junio de 2012].

19. Bingham $\mathrm{S}$. The dietary assessment of individuals; methods, accuracy, new techniques and recommendations. Nutr Abstr Rev A 1987; 57: 706-42.

20. Tanaka T, Okamura T, Miura K, Kandowaski T, Ueshima $\mathrm{H}$, Nakagawa $\mathrm{H}$, et al. A simple method to estimate populational 24-h urinary sodium and potassium excretion using a casual urine specimen. J Hum Hypertens 2002; 16 (2): 97-103.

21. Bland J, Altman D. Statistical Methods for Assessing Agreement between Two Methods of Clinical Measurement. Lancet 1986; 1 (8476): 307-10.

22. Panel on Dietary Reference Intakes for Electrolytes and Water, Standing Committe on the Scientific Evaluation of Dietary Reference Intakes. Dietary Reference Intakes for Water, Potassium, Sodium, Chloride, and Sulfate; Institute of Medicine 2005. Disponible en: http://www. nal.usda.gov/fnic/DRI/DRI_Water/water_full_report. 
Ingesta dietética y excreción urinaria de sodio y potasio - K. Cornejo et al

pdf. [Consultado el 04 de agosto de 2012].

23. World Health Organization. Reducing Salt Intake in Populations: Report of a WHO Forum and Technical Meeting, 5-7 october 2006. Disponible en: http://www. who.int/dietphysicalactivity/Salt_Report_VC_april07. pdf. [Consultado el 04 de agosto de 2012].

24. López G, Galván M, Muzzo S. Excreción urinaria de sodio en niños y adultos de una comuna de la Región Metropolitana de Santiago de Chile. Rev Chil Nutr 2009; 36 (4): 1139-43. 